\title{
An experimental evaluation of differential natural reactivity as a basis for the oscillation effect
}

\author{
JOHN GAITO \\ York University, Downsview, Ontario, M3J 1P3, Canada
}

\begin{abstract}
Previous research indicated that an oscillation effect resulted during sequential alternation of unilateral amygdaloid stimulation with consistent low-latency values for one side and consistent high values for the contralateral one. One possible basis for this effect is that one of the two sides naturally has a greater reactivity to the stimulating current. This hypothesis was evaluated in this study. One group of rats had the usual alternation of stimulation from one side to the other over 10 phases of six convulsions each. A second group received five consecutive phases of stimulation of the primary site and then five consecutive phases for the secondary side. If the hypothesis were true, the latency values for one side would be consistently lower (or higher) than those for the phases on the other side; this result did not occur, although significant oscillation patterns were prominent with the alternation group. These results tend to suggest that differential natural reactivity of the two sides is not the basis for the cscillation effect.
\end{abstract}

The "kindling effect" has been investigated in a number of laboratories as a model of learning, a model of epilepsy, or as an example of behavioral change of interest in its own right (e.g., Gaito \& Gaito, 1974; Goddard, McIntyre, \& Leech, 1969). This event involves a change from normal exploration to automatic behaviors and, finally, to clonic convulsions (CCs) in response to periodic low-intensity electrical stimulation of a specific brain site (e.g., amygdala).

In recent research in our laboratory using a sequence of alternating unilateral stimulations of the amygdalae, an "oscillation effect" was observed (Gaito, 1976). This oscillation usually consisted of low values for the amygdala first stimulated (primary site) and high values for the contralateral amygdala (secondary site). The effect was most prominent in latency data (i.e., number of seconds between onset of stimulation and onset of convulsion). The oscillation tendency in latency data has been remarkably resistant to a number of experimental manipulations (Gaito, Gaito, \& Nobrega, 1977).

There are two types of oscillation that occur consistently in our research. Primary oscillation (PO) involves low values for the primary site and high values for the secondary site for at least 8 of the 10 phases. Secondary oscillation (SO) is the opposite pattern.

One possible basis for this effect can be indicated as the differential natural reactivity hypothesis. This hypothesis would specify that one of the two sides has a greater natural reactivity to the stimulating frequency; thus, stimulation of this side would produce a quick reaction that is expressed as a low latency.

This research was supported by a grant from the President's National Research Council Fund (York University).
There was some indirect evidence for suggesting that differential natural reactivities of the two sides was not responsible for the total oscillation effect (Gaito, Nobrega, \& Gaito, 1978); however, this aspect could be a partial contributor. The purpose of the present experiment was to test this hypothesis directly.

\section{METHOD}

Twelve male Wistar rats were implanted bilaterally with bipolar electrodes in each amygdala. These rats were approximately 160 days of age. The brain coordinates for electrode implantation were the same as in previous experiments: $.5 \mathrm{~mm}$ posterior to bregma, $4.5 \mathrm{~mm}$ from midline, $8.5 \mathrm{~mm}$ from skull (Gaito, 1976; Gaito \& Gaito, 1974).

Stimulation trials began 1 week or more after electrode implantation. Each trial consisted of passing a $60-\mathrm{Hz}$ sine wave for $30 \mathrm{sec}$ into the rat, using a Lafayette stimulator. There were 3 trials/day (approximately $1 \mathrm{~h}$ apart). The initial intensity was set at 100 microA for all animals; the current was gradually increased in the cases of no CC by 10 trials until the appropriate intensity was achieved for each animal. In most cases, the convulsion was achieved with $100 \mathrm{microA}$, and in no case was the intensity level raised beyond 560 microA.

On the day after the 6th CC, stimulation began at the secondary site for six of these rats (Group 1) and continued until $6 \mathrm{CCs}$ occurred. Then stimulation at the primary site was instituted again, then at the secondary site, and so on. This sequential alternation of unilateral stimulation was continued for 10 phases ( 5 of primary site stimulation and 5 for the secondary site).

For the other six rats (Group 2), each of the first five phases of $6 \mathrm{CC}$ was from primary-site stimulation. Phases 6-10 were based on secondary-site stimulation.

For each rat in Group 1, the behavioral designation for the latency and criterion measures was that of primary oscillator, secondary oscillator, or nonoscillator. For example, to be classified as an oscillator, the rat had to show a consistent pattern of "low-high" (primary oscillation) or "high-low" (secondary oscillation) in latency and/or criterion data over 
8 or 10 of the 10 alternation phases in a sequence. As in the previous research, duration values were not analyzed.

The rats in Group 2 were classified in a similar fashion. However, the sequence was Phase $1,6,2,7,3,8,4,9,5,10$. If the differential natural reactivity hypothesis were correct, these rats would be expected to show signiricant oscillation patterns because either the primary or secondary site had lower latencies consistently.

\section{RESULTS}

The electrode assembly from two rats in Group 2 was lost during Phases 6 and 7; the data from these animals were not used.

In latency data, three of the rats in Group 1 showed a primary oscillation pattern and one had the secondary pattern. The probability of an oscillation pattern by chance is $.17 .^{1}$ The probability that four oscillators in six rats will occur by chance is less than .05 , the probability level chosen for rejection of the null hypothesis, using the binomial distribution.

Only one of the four rats in Group 2 had an oscillation pattern (secondary oscillation). This result is not significantly different from that expected by chance $(\mathrm{p}>.05)$.

In criterion data, one rat in Group 1 had a secondary pattern. No Group 2 rats showed an oscillation pattern. Neither of these results was significant statistically $(p>.05)$.

\section{DISCUSSION}

The results of this experiment tend to question the authenticity of the differential natural reactivity hypothesis. This conclusion is consistent with the results of previous experiments in which this hypothesis tended to be inconsistent with observations (Gaito et al., 1978). The behavior of a number of rats suggested that an active inhibition process was occurring during stimulation of one side.

The exact basis for the effect is not known. A number of potential contributors seem to have been excluded. These include chance aspects, differential thresholds for the two sides, differential placement of the two electrodes, and differential effectiveness of the two electrodes (Gaito et al., 1978).

Presumably, there are some inhibitory and/or facilitatory effects from the primary site to the secondary site, and vice versa, to produce the oscillation effect (McIntyre \& Goddard, 1973; Nobrega \& Gaito, 1978). A pattern appears to be set up, either primary oscillation or secondary oscillation, during Phases 1 and 2 , or by Phases 3 and 4 , and most rats continue with the pattern for the remainder of the 10 phases. In one experiment in which rats were stimulated through 50 phases, some rats showed a consistent pattern of oscillation for the 50 phases (Gaito, 1978).

The unilateral sequential alternation procedure and the resulting oscillation effect appear to be useful for obtaining information on some aspects of the events underlying the kindling effect, for example, possible differential electrical synaptic patterns related to the difference in latency values for the primary and secondary sites. Because kindling shows many parallels to learning and to epilepsy and can be considered as a model of consistent behavioral changes in response to an invariant stimulus, experimentation with the oscillation effect may have the potential for providing information relevant to brain function in general.

\section{REFERENCES}

Garro, J. An oscillation effect during sequential alternations of unilateral amygdaloid stimulations within the kindling paradigm. Physiological Psychology, 1976, 4, 303-306.

GaIto, J. The oscillation effect over long-term periods. Bulletin of the Psychonomic Society, 1978, 11, 9-12.

GaIto, J., \& Gaito, S. T. Interanimal negative transfer of the kindling effect. Physiological Psychology, 1974, 2, 379-382.

Gaito, J., Gaito, S. T., \& Nobrega, J. N. A factor analysis of data from ten phases of sequential alternations of amygdaloid stimulation within the kind ling paradigm. Physiological Psychology, 1977, 5, 300-310.

Gaito, J., Nobrega, J. N., \& Gaito, S. T. Statistical evaluation of several aspects concerning the oscillation effect. Physiological Psychology, 1978, 6, 209-214.

Goddard, G. V., McIntyre, D. C., \& Leech, C. K. A permanent change in brain function resulting from daily electrical stimulation. Experimental Neurology, 1969, 25, 295-330.

HoEl, P. G. Introduction to mathematical statistics. New York: Wiley, 1954.

MCINTYRe, D. C., \& Goddard, G. V. Transfer, interference and spontaneous recovery of convulsions kindled from the rat amygdala. Electroencephalography and Clinical Neurophysiology, 1973, 35, 533-543.

NobRega, J. N., \& Gaito, J. Long term induction of kindled seizures in rats: Interhemisphere factors. Canadian Journal of Neurological Sciences, 1978, 5, 223-230.

\section{NOTE}

1. The hypothesis that the oscillation patterns are random ones can be assessed by the one-sample runs test (Hoel, 1954). Using the requirement of 8 or 10 phases of oscillation out of 10 phases would provide 8,9 , or 10 runs. The $p$ of 8,9 , or 10 runs is $.1270, .0317$, and .0079 , respectively; the $p$ of 8 , 9 , or 10 runs is the sum of these ps, .1666 or .17. Thus, the $p$ that these apparent systematic patterns are random ones would be .17 .

(Received for publication October 9, 1978.) 